\title{
Candida intermedia
}

National Cancer Institute

\section{Source}

National Cancer Institute. Candida intermedia. NCI Thesaurus. Code C120706.

A species of pseudohyphae fungus belonging to the genus Candida that has been isolated from human skin, throat, and feces; it is a rare human pathogen in nosocomial infections. 